\title{
A Novel Low Recurrence Surgical Approach for Medulloblastoma Excision: A Study of 58 Cases
}

\author{
Nitin DANGE ${ }^{1}$, Nilesh BAKALE ${ }^{1}$, Aniruddh AGRAWAL ${ }^{2}$ \\ ${ }^{1}$ King Edward Memorial Medical College and Hospital, Department of Neurosurgery, Mumbai, India \\ ${ }^{2}$ Topiwala National Medical College, Mumbai, India \\ Corresponding author: Nitin N. DANGE docdnitin@yahoo.com
}

\section{ABSTRACT}

AIM: To describe a novel surgical approach to decrease the complication and recurrence rates of medulloblastomas.

MATERIAL and METHODS: A retrospective review was performed. The novel technique was performed under similar conditions for all patients. The technique involved early intra-operative identification of the superior part of the floor of the fourth ventricle, so that the inferior part of the tumor could be viewed directly and excised thoroughly. Importance was given to the pattern of tumor growth and CSF flow dynamics.

RESULTS: A total of 58 patients underwent this surgery between February-2006 and May-2016. Mean age was 13.2 years (Range - 6 months to 55 years). Follow-up ranged from 1 to 11 years. 49 patients (84.4\%) who were under the age of 3 years were administered craniospinal radiation as well as chemotherapy. 9 patients (15.6\%) over the age of 3 years were administered only chemotherapy.

Total excision could be performed in 50 cases (86.2\%) and subtotal excision (>90\% excision) in 8 cases (13.8\%). The tumor recurred in only 1 patient $(1.72 \%)$. The complications included akinetic mutism $(8.6 \%)$, meningitis $(8.6 \%), 6^{\text {th }} \& 7^{\text {th }}$ nerve paresis/palsy $(5.17 \%)$, and chest infection (3.44\%). Death occurred in 2 patients $(3.44 \%)$, causes of death were unrelated to surgery.

CONCLUSION: This study highlights the possible benefits of this novel approach to medulloblastoma excision by decreasing recurrence and complication rates and increasing rates of total excision. However, studies with large cohorts need to be performed to evaluate its efficacy.

KEYWORDS: Medulloblastoma, Recurrence, Surgery, Excision

\section{INTRODUCTION}

$\mathrm{D}$ iscovered by Cushing and Bailey in 1925, medulloblastomas (MB) are common malignant tumors of the cerebellum. They are the most common posterior fossa tumors in children (3). Approximately $85 \%$ of medulloblastoma are located in the cerebellar midline, typically arising from the inferior medullary velum and expanding into the fourth ventricle and/or cisterna magna. These tumors often extend inferiorly and laterally (12). The remaining $15 \%$ present as lateral masses located in the cerebellar hemisphere or the cerebellopontine angle (10). The anatomy and neurosurgical relevance of these regions have been researched extensively $(14,22)$.
Surgical treatment remains a mainstay in the management of medulloblastomas $(9,11)$. The two most common surgical approaches to exposing the medulloblastoma in the fourth ventricle are the telovelar and the transvermian approaches (20). The telovelar approach involves dissecting the cerebellomedullary fissure, the opening of the tela choroidea, and the inferior medullary velum, followed by superior retraction of the lower vermis. In the transvermian approach, the inferior vermis of the cerebellum is divided, and the two halves of the vermis are retracted in two opposite directions. The telovelar approach is usually preferable for all fourth ventricle tumors; however, in large tumors, this technique is considered hazardous since it involves higher complication 
rates (16). The telovelar approach is also limited in approaching cranial parts of large tumors (21). However, both surgical techniques require extensive dissection in a neurologically eloquent area. The transvermian approach requires an incision into the cortical and functional regions of the cerebellum and splitting the inferior cerebellar vermis, which may give rise to the caudal vermis syndrome or cerebral mutism (5).

Apart from excessive dissection, these surgical procedures also have high recurrence rates in medulloblastomas, which range from $26 \%$ to $32 \%(13,23)$. The major complication occurrence rate ranges from $11 \%$ to $29 \%$ (15). Postoperative complications include hydrocephalus, pneumocephalus, hematoma, aseptic meningitis, gastrointestinal hemorrhage, cervical instability, neurological deficits (secondary to damage of the cerebellum, brain stem, and cranial nerves) such as truncal ataxia and hypotonia (lesions of the anterior lobe of the cerebellum), appendicular ataxia, hypotonia, tremor (lesions of the posterior lobe), hemiparesis, and lower cranial nerve dysfunction (brainstem injury).

These varying and grim complications, coupled with high mortality, morbidity, and recurrence rates, called for an alternative technique that involved less surgical dissection in the eloquent area and minimal damage to the cerebellum and other vital structures. Using this technique would potentially translate to fewer postoperative complications. Total and subtotal resections (residual tumor $<1.5 \mathrm{cc}$ ) have a significantly lower chance of recurrence (1), so it was also important to decrease damage to surrounding areas and increase the probability of subtotal and total resection of the tumor. The authors, therefore, went on to establish and describe a modified approach to a fourth-ventricle medulloblastoma with limited dissection and negligible recurrence rates.

\section{MATERIAL and METHODS}

This study was a retrospective analysis of a prospective database of 58 patients diagnosed with medulloblastoma presenting at a tertiary care hospital between February 2006 and May 2016. All patients with Horseradish Peroxidase (HPR) suggestive of medulloblastoma were included; no case was excluded from the cohort. None of the patients had primary tumor metastasis.

The same surgeon operated on all the patients with the same surgical approach. A subtotal resection was defined as resection of more than $90 \%$ of the tumor or a residual tumor $(<1.5 \mathrm{cc})$ on postoperative imaging. Radiation and chemotherapy protocols, follow-up neuroimaging, and metastatic workup were reviewed.

\section{Surgical Technique}

The authors describe their technique for safe radical excision of a fourth ventricle medulloblastoma depending upon its origin, the direction of growth, and natural plane maintained due to the downward resistance offered by CSF to their growth. Figure 1 describes the $4^{\text {th }}$ ventricle anatomy.

The authors believe the positioning of the patient to be a crucial detail while attempting this technique. A sitting position was preferred by the author, although it is a double-edged sword. Based on experience, it offers added exposure and improved blood and CSF drainage from the operative field but also increases the risk of an air embolism, postoperative pneumocephalus as well as tearing of the bridging veins between the cerebellum and the tentorium (2).

Midline skin incision, extending from inion to the C2 spinous process, was adequate. Skin flaps were mobilized. The cervical musculature was also mobilized laterally by sharp dissection of the occipital bone in the avascular plane. The foramen magnum was then exposed. Midline suboccipital craniectomy was performed by placing burr-holes below the transverse sinuses. The dura mater was opened in a " $U$ " shaped manner, followed by a thorough inspection of exposed cerebellar to rule out leptomeningeal seeding.

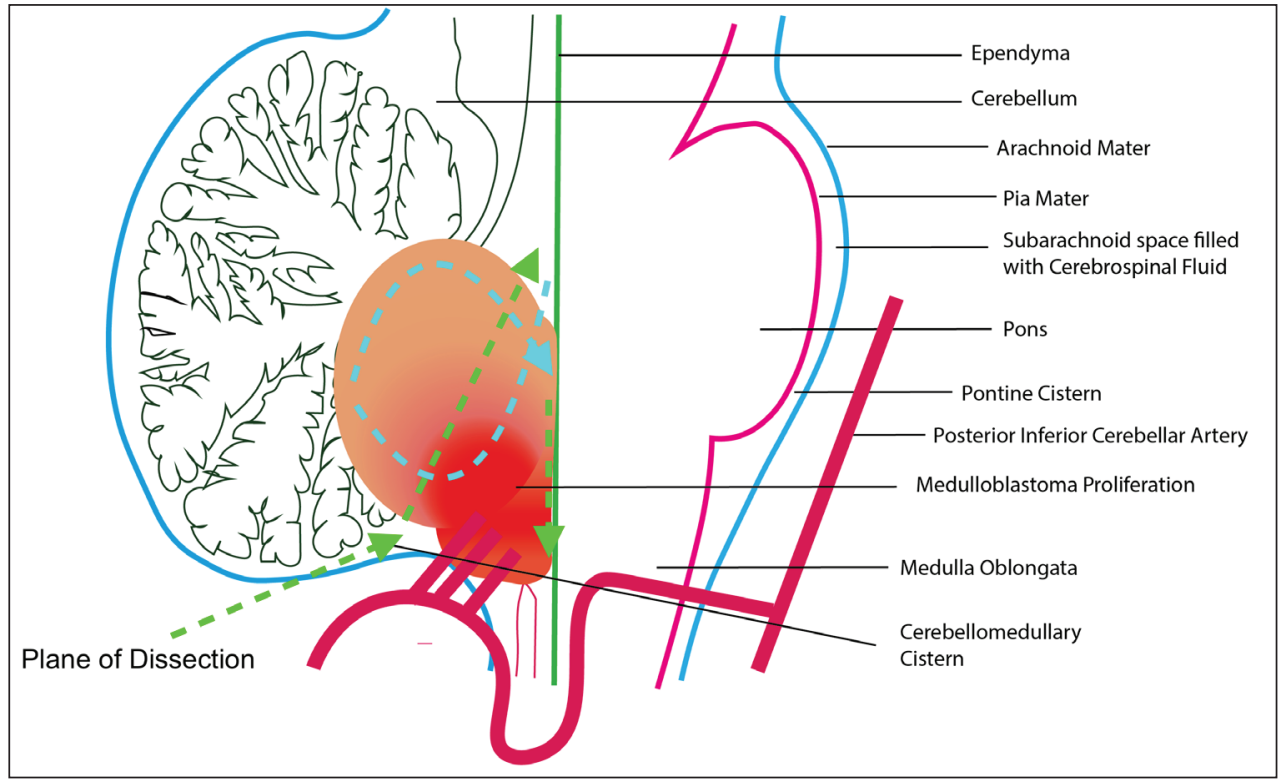

Figure 1: Anatomy of the fourth ventricle. 


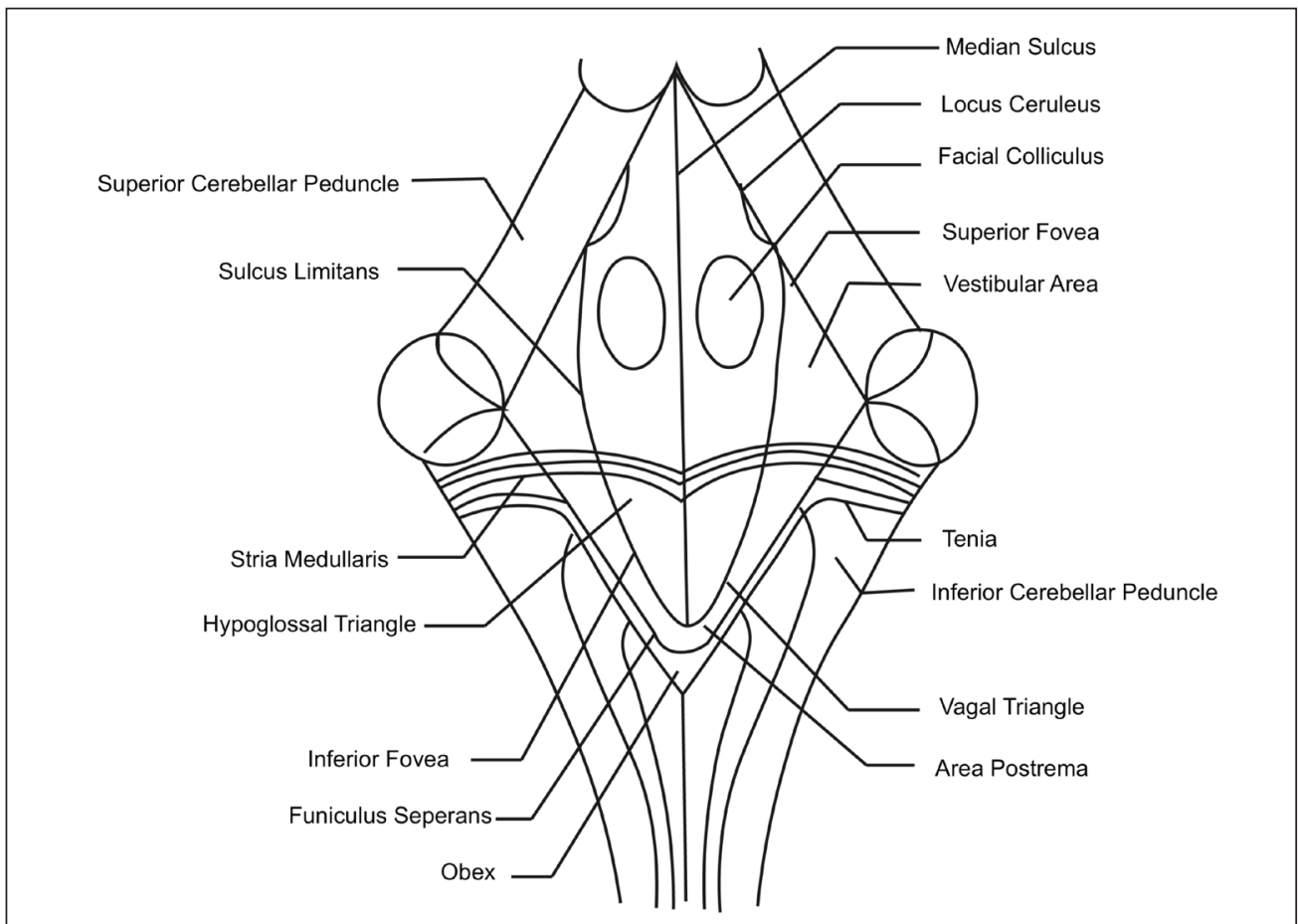

Figure 2: The Approach to the medulloblastoma. Note that once the cerbellum is retracted to expose the cerebello-medullary sulcus, we enter the tumor and dissection is directed antero superiorly till we reach the floor of fourth ventricle. After the vital brainstem structures are identified separate from the tumor, the tumor is debulked. Also note this portion of tumor is soft, easily suckable and less vascular. Later the inferior portion of the tumor i.e. hard and more vascular.

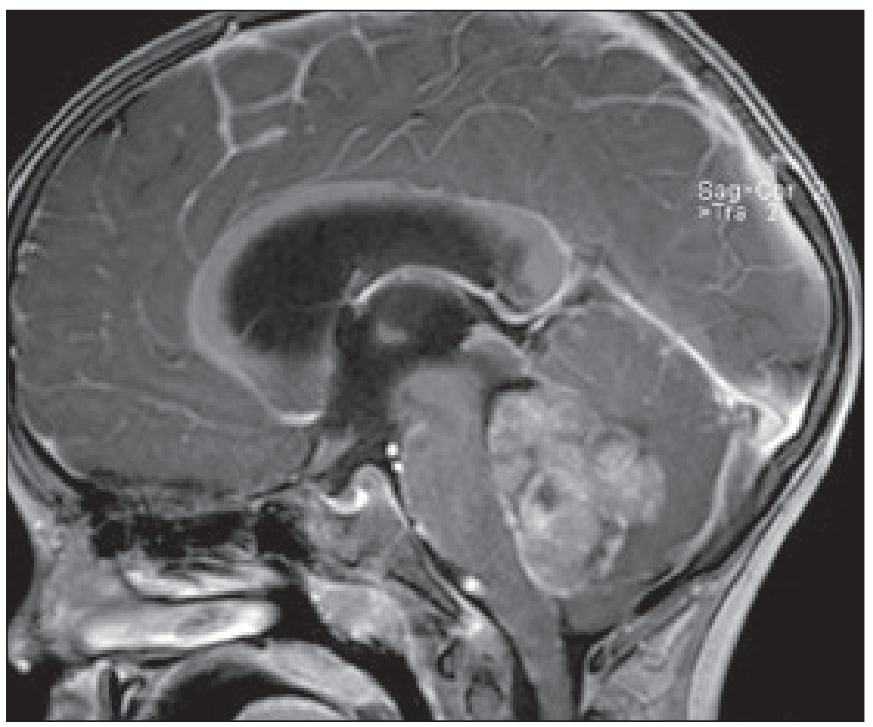

Figure 3: Images of MRI of brain of patients with medulloblastoma (sagittal view). Note the presence of CSF space just anterior to the tumor separating it from the floor of fourth ventricle. This was used to track the plane of dissection, and minimize brainstem injuries and other complications.

The vermis was then retracted rostrally to expose the tumor at the lower end of the fourth ventricle floor and approach it from its posterior cerebellar surface. Once inside the tumor, the authors proceeded in the anterosuperior direction until the fourth ventricle floor was exposed. Once the fourth ventricle floor was identified, the plane was then traced downwards between the brainstem and tumor. The tumor was then excised, to the extent possible, in a piece-meal manner as usual. If the tumor adhered to the fourth ventricle floor, then the tumor mass was resected in the floor plane. Figure 2 is a pictorial representation of the surgical technique, and Figure 3 shows the location of the medulloblastoma in the fourth ventricle.

Since the floor of the fourth ventricle was identified early in this approach and traced caudally, the chances of injuring the neurological structures, especially the cranial nerve nuclei and brain stem, were minimized.

\section{Permissions and Analyses}

All statistical analyses were performed with SPSS v24 software. Means and standard deviations were used to describe continuous variables. A statistician who was not involved in the surgical treatment of the patients analyzed the data.

All requisite permissions from the Institutional Review Board were obtained before the commencement of this project. All patients provided informed consent before each surgery that the data from their procedure could be used for research purposes and possible publication.

\section{RESULTS}

\section{Patient Demographics}

The database consisted of 58 patients diagnosed with medulloblastoma treated between February 2006 and May 2016. Mean age: 13.16 years. (range 6 months-55 years). There were 35 male patients and 23 female patients. Sex Ratio (Male: Female) was 1.52: 1. Table I highlights these demographic details. 
Table I: Showcases the Demographic Details of the Patients as well as the Complication Rates [N(\%)] for All Patients. It Also Gives an Indication of How Many Patients Received Radiotherapy and Chemotherapy and How Many Received Only Chemotherapy

\begin{tabular}{|c|c|c|}
\hline & & Patients n(\%) \\
\hline \multirow{2}{*}{ Sex } & Male & 35 (60.34) \\
\hline & Female & 23 (39.65) \\
\hline \multirow{2}{*}{$\begin{array}{l}\text { Surgery } \\
\text { Performed }\end{array}$} & Total Excision & 50 (86.20) \\
\hline & Subtotal Excision & $8(13.79)$ \\
\hline \multirow{2}{*}{$\begin{array}{l}\text { Adjuvant } \\
\text { Therapy }\end{array}$} & $\begin{array}{c}\text { Radiotherapy and } \\
\text { chemotherapy }\end{array}$ & $49(84.4)$ \\
\hline & Chemotherapy only & $9(15.6)$ \\
\hline \multirow{7}{*}{ Complications } & Akinetic Mutism & 5 (8.62) \\
\hline & Nerve Paresis $\left(6^{\text {th }} \& 7^{\text {th }}\right)$ & $3(5.17)$ \\
\hline & Meningitis & $5(8.62)$ \\
\hline & Chest Infection & $2(3.44)$ \\
\hline & Recurrence & $1(1.72)$ \\
\hline & Death & $2(3.44)$ \\
\hline & No complications & $40(68.96)$ \\
\hline
\end{tabular}

In all patients, the tumor was located in the midline and expanded into the fourth ventricle floor. All patients underwent surgical excision using the technique above.

Patients over 3 years of age (84.4\%) received craniospinal radiation and adjuvant chemotherapy (Cisplatin and Vincristine based). Those under 3 years of age (15.6\%) received chemotherapy only. All patients from the database are included in the study.

\section{Outcome Measures}

Patients were followed up postoperatively. The mean follow-up was six years (Range 1 year-11 years). No patient had primary tumor metastasis. They were evaluated for the presence of complications, recurrence, and any significant clinical changes. Recurrence was noted on Magnetic Resonance Imaging (MRI) in one patient. All patients underwent MRI scanning 72 hours postoperatively to determine the degree of resection obtained. These images were reviewed by a consultant neuroradiologist, the treating surgeon, and the treating oncologist. A consensus was then reached about the degree of resection. In 50 cases, total excision could be achieved. In 8 cases, only subtotal excision could be done due to the infiltration of the medulla and cerebellar peduncles. In these cases, too, the residual tumor was significantly less $\left(<1.5 \mathrm{cc}^{3}\right)$. There were two mortalities (3.45\%). In both cases, the cause of death was not related to this surgery or the recurrence of tumors, as noted by the physician treating them at the time. The deaths were both related to cardiovascular issues at least three years after the surgery was performed.

Recurrence was noted in just one patient (1.72\%).

Complications from the surgery observed included akinetic mutism in $5(8.62 \%)$ cases, which spontaneously improved. 3 $(5.17 \%)$ cases had developed $6^{\text {th }} \& 7^{\text {th }}$ nerve paresis. 5 cases (8.62\%) developed meningitis, and 2 cases (3.45\%) developed a chest infection.

\section{DISCUSSION}

Medulloblastomas arise from the fourth ventricle roof, which is formed by the inferior medullary velum. Most of the medulloblastomas are anterior vermian in location. These tumors expand in the fourth ventricle cavity and grow toward the floor of the fourth ventricle (17). Because of the continuous downward push by CSF flowing down through the aqueduct, there is a persistent resistance faced by the growing tumor. The resistance of the CSF flow offered to the tumor creates a surgical plane between the tumor and the superior aspect of the fourth ventricle floor. CSF flow through the aqueduct into the fourth ventricle keeps the tumor away from the fourth ventricle floor at the superior aspect. In our technique, we used this plane to our advantage. The plane is identified early in the operation, and it is maintained to minimize injury to eloquent areas, such as the brainstem. The lower part of the fourth ventricle is the earliest to be infiltrated by the tumor. Therefore, if the surgeon proceeds anteriorly, the probability of entering into the brainstem is high. In our technique, the tumor can be safely excised under the vision and radically resected from the floor of the fourth ventricle without any injury to the fourth ventricular floor. The thin layer of tumor adherent to the lower part of the fourth ventricular floor has to be tackled at the end of surgery.

Another advantage offered by this novel approach is that we deal with the superior and anterior parts of the tumor earlier in the surgery. These parts are relatively avascular and are away from the proliferating central part of the tumor. Hence, these parts are soft and can be easily aspirated with a suction cannula and then excised with minimal blood loss. The firm and vascular part is dealt with once eloquent structures are identified. The most inferior and relatively more difficult to dissect part is handled at the end when the brainstem is visible; therefore, it can be easily dissected from the tumor, reducing the risk of further injury. In our series also, we can see that most of the patients underwent complete excision, and only eight patients had subtotal/near-total excision. In these subtotal excision cases, the remaining tumor was significantly small in size $(<1.5 \mathrm{cc})$.

The value of radical excision of medulloblastoma was emphasized as early as in 1930 by Cushing (4). Aggressive surgery without compromising function provides pathological diagnosis and reestablishes the CSF pathway. Whenever possible, a shunt should be avoided to close a potential portal for extra-neural metastasis (6). Gross total resection strongly correlated with improved survival in children under 3 (19).

As per classical approaches, $8 \%-38 \%$ of patients with midline cerebellar medulloblastoma suffer from postoperative posterior fossa mutism syndrome, which typically occurs in younger patients with high-risk disease. Mutism develops over 48-72 hours after tumor resection and can persist from weeks to months, with associated findings of dysmetria, hypotonia, dysphagia, hemiparesis, and increased mood lability. Up to $50 \%$ of patients have long term speech and language apraxia. Preoperative brainstem invasion was the only feature associated with a risk of posterior fossa mutism syndrome. The proposed neurophysiological mechanism is 
Dange N. et al: Novel Approach to Medulloblastoma Excision

the disruption of the dentate-rubro-thalamic pathways to the supplementary motor cortex, secondary to surgically induced vermian damage, leading to global akinesia and speech apraxia (18).

A study performed on the telovelar approach's efficacy for medulloblastoma resection (8) showed a higher degree of complications like akinetic mutism (13.6\%), recurrence (6.8\%), and even death. Furthermore, the proportion of cases that could achieve only subtotal resection cases were higher in this cohort of patients (29.2\%). Another study with three patients (7) of medulloblastoma, resected using the telovelar approach, showed akinetic mutism in $2(66.6 \%)$ of its cases (7).

This new technique we are presenting showed decreased rates of akinetic mutism (8.62\%), recurrence $(1.72 \%)$, and a substantially higher percentage of individuals in whom total resection could be achieved (86.20\%).

\section{CONCLUSION}

The approach described by authors is the identification of the fourth ventricle floor early in surgery and then debulking of tumor supero-inferiorly with the fourth ventricle floor under vision throughout the surgery. This approach poses less risk of injury to the floor of the fourth ventricle. Although radical surgical excision of the tumor remains a mainstay, it is important to evaluate chemotherapeutic, radiotherapeutic, and novel oncogenetic therapies in the treatment of medulloblastomas.

\section{ACKNOWLEDGEMENTS}

The authors would like to thank Prof. Atul Goel, M.D., at Department of Neurosurgery, KEM Hospital and Seth G S Medical College, Mumbai.

The authors would like to thank ENAGO (www.enago.com) for the English language review.

\section{REFERENCES}

1. Albright AL, Wisoff JH, Zeltzer PM, Boyett JM, Rorke LB, Stanley P: Effects of medulloblastoma resections on outcome in children: A report from the Children's Cancer Group. Neurosurgery 38:265-271, 1996

2. Atallah A, Rady MR, Kamal HM, El-Mansy N, Alsawy MFM, Hegazy A, Zaghloul M: Telovelar Approach to Pediatric Fourth Ventricle Tumors: Feasibility and Outcome. Turk Neurosurg 29:497-505, 2019

3. Bailey $\mathrm{P}$, Cushing $\mathrm{H}$ : Medulloblastoma cerebelli: A common type of midcerebellar glioma of childhood. Archives of Neurology \& Psychiatry 14:192-224, 1925

4. Cushing $\mathrm{H}$ : Experiences with the cerebellar medulloblastomas. Acta Pathologica Microbiologica Scandinavica 7:1-86, 1930

5. Dailey AT, McKhann GM, Berger MS: The pathophysiology of oral pharyngeal apraxia and mutism following posterior fossa tumor resection in children. J Neurosurg 83:467-475, 1995

6. Due-Tønnessen BJ, Helseth E: Management of hydrocephalus in children with posterior fossa tumors: Role of tumor surgery. Pediatr Neurosurg 43:92-96, 2007
7. El-Bahy K: Telovelar approach to the fourth ventricle: Operative findings and results in 16 cases. Acta Neurochir (Wien) 147:137-142; discussion 142, 2005

8. Geldsetzer P: Use of rapid online surveys to assess people's perceptions during infectious disease outbreaks: A crosssectional survey on COVID-19. J Med Internet Res 22:e18790, 2020

9. Gupta T, Sarkar C, Rajshekhar V, Chatterjee S, Shirsat N, Muzumdar D, Pungavkar S, Chinnaswamy G, Jalali R: Indian Society of Neuro-Oncology consensus guidelines for the contemporary management of medulloblastoma. Neurol India 65:315-332, 2017

10. Maleci A, Cervoni L, Delfini R: Medulloblastoma in children and in adults: A comparative study. Acta Neurochir (Wien) 119:62-67, 1992

11. Martin AM, Raabe E, Eberhart C, Cohen KJ: Management of pediatric and adult patients with medulloblastoma. Curr Treat Options Oncol 15:581-594, 2014

12. Matsushima T: Microsurgical Anatomy and Surgery of the Posterior Cranial Fossa. Japan: Springer, 2015

13. Modha A, Vassilyadi M, George A, Kuehn S, Hsu E, Ventureyra EC: Medulloblastoma in children-the Ottawa experience. Child's Nervous System 16:341-350, 2000

14. Mussi AC, Rhoton AL: Telovelar approach to the fourth ventricle: microsurgical anatomy. J Neurosurg 92:812-823, 2000

15. Spennato P, Ruggiero C, Cinalli G: Medulloblastoma - surgery. In: Özek MM, Cinalli G, Maixner W, Sainte-Rose C (eds), Posterior Fossa Tumors In Children. Switzerland: Springer, 2015:313-332

16. Rajesh BJ, Rao BR, Menon G, Abraham M, Easwer HV, Nair S: Telovelar approach: Technical issues for large fourth ventricle tumors. Child's Nervous System 23:555-558, 2007

17. Raybaud C, Ramaswamy V, Taylor MD, Laughlin S: Posterior fossa tumors in children: Developmental anatomy and diagnostic imaging. Child's Nervous System 31:1661-1676, 2015

18. Robertson PL, Muraszko KM, Holmes EJ, Sposto R, Packer RJ, Gajjar A, Dias MS, Allen JC, Group CsO: Incidence and severity of postoperative cerebellar mutism syndrome in children with medulloblastoma: A prospective study by the Children's Oncology Group. J Neurosurg 105:444-451, 2006

19. Rutkowski S, von Bueren A, von Hoff K, Hartmann W, Shalaby T, Deinlein F, Warmuth-Metz M, Soerensen N, Emser A, Bode U, Mittler U, Urban C, Benesch M, Kortmann RD, Schlegel PG, Kuehl J, Pietsch T, Grotzer M: Prognostic relevance of clinical and biological risk factors in childhood medulloblastoma: Results of patients treated in the prospective multicenter trial HIT'91. Clin Cancer Res 13:2651-2657, 2007

20. Tanriover N, UIm AJ, Rhoton AL Jr, Yasuda A: Comparison of the transvermian and telovelar approaches to the fourth ventricle. J Neurosurg 101:484-498, 2004

21. Tomasello F, Conti A, Cardali S, La Torre D, Angileri FF: Telovelar approach to fourth ventricle tumors: Highlights and limitations. World Neurosurgery 83:1141-1147, 2015

22. Tubbs RS, Bosmia AN, Loukas M, Hattab EM, Cohen-Gadol AA: The inferior medullary velum: Anatomical study and neurosurgical relevance. J Neurosurg 118:315-318, 2013

23. Wong TT, Liu YL, Ho DM, Chang KP, Liang ML, Chen HH, Lee YY, Chang FC, Lin SC, Hsu TR, Chen KW, Kwang WK, Hou WY, Wang CY, Yen SH, Guo WY, Chen YW: Factors affecting survival of medulloblastoma in children: The changing concept of management. Child's Nervous System 31:1687-1698, 2015 